\title{
Systematic study of magnetar-powered hydrogen-rich supernovae
}

\author{
Mariana Orellana $^{1, \star}$, Melina C. Bersten ${ }^{2,3,4}$, and Takashi J. Moriya ${ }^{5}$ \\ ${ }^{1}$ Laboratorio de Procesamiento de Señales Aplicado y Computación de Alto Rendimiento, Sede Andina, Universidad Nacional de \\ Río Negro, Mitre 630, 8400 Bariloche, Argentina \\ e-mail: morellana@unrn.edu.ar \\ 2 Instituto de Astrofísica de La Plata (IALP), CCT-CONICET-UNLP, Paseo del Bosque s/n, La Plata B1900FWA, Argentina \\ ${ }^{3}$ Kavli Institute for the Physics and Mathematics of the Universe (WPI), The University of Tokyo, 5-1-5 Kashiwanoha, Kashiwa, \\ Chiba 277-8583, Japan \\ ${ }^{4}$ Facultad de Ciencias Astronómicas y Geofísicas, Universidad Nacional de La Plata, Paseo del Bosque s/n, \\ B1900FWA La Plata, Argentina \\ 5 Division of Theoretical Astronomy, National Astronomical Observatory of Japan, National Institutes of Natural Sciences, 2-21-1 \\ Osawa, Mitaka, Tokyo 181-8588, Japan
}

Received 18 January 2018 / Accepted 31 August 2018

\begin{abstract}
Context. It has been suggested that some supernovae ( $\mathrm{SNe}$ ) may be powered by a magnetar formed at the moment of the explosion. While this scenario has mostly been applied to hydrogen-free events, it may also be possible for hydrogen-rich objects.

Aims. We aim to explore the effect of including a magnetar on the light curves of supernovae with H-rich progenitors.

Methods. We have applied a version of our one-dimensional local thermodynamic equilibrium radiation hydrodynamics code that takes into account the relativistic motion of the ejecta caused by the extra energy provided by the magnetar. For a fixed red supergiant (RSG) progenitor, we have obtained a set of light curves that corresponds to different values of the magnetar initial rotation energy and the spin-down timescale. The model is applied to SN 2004em and OGLE-2014-SN-073, two peculiar Type II SNe with long-rising SN 1987A-like light curves, although with much larger luminosities.

Results. The presence of a plateau phase in either normal or superluminous supernovae is one possible outcome, even if a magnetar is continuously injecting energy into the ejecta. In other cases, the light curve shows a peak but not a plateau. Also, there are intermediate events with a first peak followed by a slow decline and a late break of the declining slope. Our models show that bright and long rising morphologies are possible even assuming RSG structures.

Conclusions. A large number of supernova discoveries per year reveal unexpected new types of explosions. According to our results, SLSNe II-P are to be expected, as well as a variety of light curve morphologies that can all be possible signs of a newly born magnetar.
\end{abstract}

Key words. supernovae: general - supernovae: individual: OGLE-2014-SN-073 - supernovae: individual: SN 2004em supernovae: individual: peculiar-87A

\section{Introduction}

Nowadays, supernovae ( $\mathrm{SNe}$ ) are known to be varied phenomena. Their classification has evolved in parallel to the increasing volume of photometric and spectroscopic data and the long standing efforts to explain their nature. Superluminous $\mathrm{SNe}$ (SLSNe), discovered already more than a decade ago, reach luminosities of ten to 100 times larger than regular $\mathrm{SNe}$ (Quimby et al. 2011; Gal-Yam 2012). They present stimulating cases to explore the extents of the available theoretical models. One of the main proposals to provide the extra source powering the luminosity of SLSNe is the formation of a magnetar. The rotational energy of the hypothetical magnetar would be responsible for the extra energy needed to power the very bright light curve (LC). Although the magnetar model has been used previously in the literature (see e.g., Maeda et al. 2007, for the peculiar SN 2005bf), it became more popular after the works of Woosley (2010) and Kasen \& Bildsten (2010). These authors showed that, if a rapidly rotating (millisecond period initially) neutron star with a large magnetic field $\left(B \sim 10^{14} \mathrm{G}\right)$ is assumed to fully deposit its energy in the ejecta, the resulting SN may

\footnotetext{
* Member of the Carrera del Investigador Científico, CONICET, Argentina.
}

reach a peak luminosity in excess of $\simeq 10^{44} \mathrm{erg} \mathrm{s}^{-1}$. After this suggestion, the magnetar model was extensively used in the literature to explain several observed SNe. In particular, the semianalytic prescription by Kasen \& Bildsten (2010) has become common thanks to its relative success to reproduce the morphology of the LCs (see e.g., Inserra et al. 2013). Yet, such a treatment neglects both the formation and the expansion of the shock wave. We refer to Yu et al. (2017) or Nicholl et al. (2017) for recent statistical studies applying this simple model.

Sukhbold \& Woosley (2016) discuss the upper bounds to the energy that can be radiated by the different scenarios invoked in the literature. The most extreme cases can be explained by magnetars, though the details of the interaction between this energy reservoir and the rest of the stellar structure are not well established at the scale of the neutron star. Magnetar power has been mainly proposed as a possible central source for $\mathrm{H}$-free SLSNe (or SLSNe I), while interaction with the circumstellar medium (CSM) is the preferred model to explain hydrogenrich SLSNe (or SLSNe II; Chevalier \& Irwin 2011; Moriya et al. 2013). The reason for this is that most observed SLSNe II are Type IIn, (objects that show narrow lines in their spectra). This is indicative of interaction between the SN ejecta and a dense CSM (e.g., as in SN 2006gy, Smith et al. 2007). However, there are a few cases lacking the narrow and intermediate-width line 
emission, such as SN 2008es, which was an H-rich non-Type IIn (Miller et al. 2009; Gezari et al. 2009, see also Inserra et al. 2018). Some other SLSNe were initially H-poor but $\mathrm{H} \alpha$ emission was later found (Yan et al. 2017). It could be possible that some of these objects were powered by a magnetar source.

In addition to SLSNe I, magnetar models have been used to explain other peculiar objects, such as the unusual SN 2005bf (Folatelli et al. 2006; Maeda et al. 2007) and its recent analog SN PTF11mnb (Taddia et al. 2018).

Many efforts have been made to deal with magnetism in SN explosions (Hu \& Lou 2009). Current knowledge indicates that progenitors with fast-rotating iron cores likely develop magnetorotational instabilities (e.g., Akiyama et al. 2003; Heger et al. 2005) as part of the mechanism that increases the magnetic field strength (Mösta et al. 2015). Simulations suggest that magnetorotational explosions could be asymmetric. Burrows et al. (2007) analyze the dynamical effects of magnetic stresses on the $\mathrm{SN}$, along with the possible jet formation that connects $\mathrm{SNe}$ with gamma-ray bursts (see also Wheeler et al. 2000). Recent works also discuss how possible jets launched at the birth of the magnetar cannot be ignored during the explosion itself nor later when fall-back mass accretion might occur (Soker \& Gilkis 2017). SN explosions might be asymmetric when influenced by a powerful magnetar. In that case, the $1 \mathrm{D}$ approach is certainly unrealistic. Chen et al. (2016) study the dynamical effect of the magnetar energy deposition based on 2D simulations. Although radiation transport is neglected, that work shows that fluid instabilities cause strong mixing and fracture shells of ejecta into filamentary structures which could affect photon emission. There are many issues related to the formation and deposition of the magnetar energy that remain unclear, although these are beyond the scope of the present study.

In the context of H-rich progenitors, magnetar-powered LCs have not been deeply studied in the literature. Bersten \& Benvenuto (2016) present a tentative simulation for a red supergiant (RSG) progenitor showing that, as expected for this type of progenitor, the plateau phase is still present in some cases when a magnetar source is taken into account. More recently, Sukhbold \& Thompson (2017) and Dessart \& Audit (2018) discuss similar scenarios, the former focused on on ordinary Type II-P SNe and the latter on SLSNe. In this work we analyze whether both cases can be embraced by variations of the magnetar characteristics.

The paper is organized as follows. Our calculations were performed with the code described in Bersten et al. (2011). In Sect. 2 we explain the modifications that we introduced in the code in order to treat this problem. The effect of magnetar parameters on the LC shape is discussed in Sect. 3, where we present our systematic analysis as a natural extension of our previous studies. In Sect. 5 we apply this model to the peculiar SN 1987Alike bright SN OGLE-2014-SN-073 (hereafter OGLE14-073), recently published by Terreran et al. (2017). The H-rich magnetar model can be applied to explain this interesting source that is one of the brightest SNe II ever discovered. We also devote a tentative parameter exploration applied to SN 2004em, another peculiar SN 1987A-like object. Discussion and conclusions including comparisons with previous works are presented in Sect. 6.

\section{Numerical model}

The inclusion of a magnetar source in our one-dimensional hydrodynamical code was recently implemented in Bersten et al. (2016). The main difference in the current work is the progenitor structure used as initial condition of the calculations. We are now interested in analyzing the possible effect of a magnetar in $\mathrm{H}$-rich objects. Therefore, we assumed a RSG structure with a thick H-envelope, typical of Type II-P SN progenitors. Our code self-consistently follows the whole evolution of the SN explosion starting from a given pre-SN structure in hydrostatic equilibrium, that is, the shock wave propagation in the stellar interior, the shock breakout, and the subsequent expansion phases. The explosion is simulated by artificially injecting thermal energy near the center of the progenitor star, without specification of the involved mechanism. A few seconds later, after the neutron star (NS) is already formed, an extra source of energy due to the magnetar is incorporated. The code assumes flux-limited radiation diffusion for optical photons and a one-group approximation for the non-local deposition of gamma-rays produced by radioactive decay of ${ }^{56} \mathrm{Ni}$ (for further detail see Bersten et al. 2011).

To parameterize the magnetar source we used a spin-down timescale $\left(t_{p}\right)$ and an initial rotation energy $\left(E_{\text {rot }}\right)$ as the free parameters of the model. They enter into the basic expression for the energy supplied per unit time by the magnetar as

$L(t)=\frac{E_{\mathrm{rot}}}{t_{p}}\left(1+\frac{t}{t_{p}}\right)^{-2}$.

These alternative parameters are equivalent to the usual $B$ (magnetic field) and $P$ (initial rotation period), but in this way we avoided including explicit properties of the NS, such as the radius or the moment of inertia, which might be explored subsequently by assuming a specific equation of state (see Bersten et al. 2016, for more details). Although the presence of a strong magnetic field in the NS interior and its coupling with matter is not fully understood, studies of the cooling of magnetized NSs (e.g., Turolla et al. 2015) have shown that the initial $B$ value is preserved for at least a few thousand years. Thus, the magnetars known today were born spinning very fast, but with a similar magnetic field to their current extreme value $B \geq 10^{13}$ Gauss.

Our strong assumption is that $L(t)$ is fully deposited and thermalized in the inner layers of the exploding star as a persistent energy injection. Specifically, we deposited the magnetar energy in the inner 15 zones of the progenitor model assuming a box function in mass coordinate. Full deposition is usually assumed in the literature, although the option of inefficient heating by the nascent magnetar was explored by Kasen et al. (2016) in order to obtain a double peaked LC. Also, the leakage of hard emission was discussed by Wang et al. (2016) as an interesting alternative to full energy trapping.

In our treatment, if the photosphere recedes deep enough for the magnetar energy to be deposited at optically thin layers, we added the magnetar contribution to the bolometric luminosity. Although the power engine is located deep into the ejecta, its influence propagates outwards pushing the lightweight outer shells up to enormous velocities. In some cases, this can lead to relativistic movements, specially in extreme cases where the energy injected by the magnetar is several orders of magnitude larger than the explosion energy, as we showed in Bersten et al. (2016). Therefore, we have modified our code to take this effect into account. In Appendix A we present the formulation of the relativistic hydrodynamics included in our 1D code.

The pre-SN models adopted throughout this work were calculated by Nomoto \& Hashimoto (1988) following the stellar evolution until core collapse. Specifically, RSG progenitors with masses of 15 and $25 M_{\odot}$ are used in this study. These stellar models assume solar metallicity and no rotation. However, low-metallicity and rotating stars are probably more realistic 
progenitors of rapidly rotating and strongly magnetized NSs than our pre-SN models. Although this is a caveat in our analysis, we note that magnetism and rotation in massive stars are complex problems for which there is still no definitive solution (Heger et al. 2003, 2005).

\section{Exploration of the parameter space}

Preliminary results of the magnetar effects in H-rich progenitors are presented in Bersten \& Benvenuto (2016). That study clearly shows that the plateau morphology of the LCs can be preserved in some cases.

In this section we consider a fixed progenitor star with mainsequence mass of $15 M_{\odot}$, pre-explosion radius of $500 R_{\odot}$, and surface metallicity of $Z \sim 0.02$. This pre-SN model shows a transition between $\mathrm{H}$-rich to He-rich layers at $\approx 3.2 M_{\odot}$. Further detail on chemical abundances can be seen in Appendix B, and a summary table with futher progenitor properties is later presented in Sect. 6. First, we discuss this reference model (Sect. 3.1) and then we focus on a grid of models (Sect. 3.2).

\subsection{Comparison: model with and without magnetar}

Figure 1 shows a comparison between models with and without a magnetar source for the progenitor star described above. We further adopted an explosion energy of 1.5 foe $\left(1\right.$ foe $\left.=1 \times 10^{51} \mathrm{erg}\right)$ and a ${ }^{56} \mathrm{Ni}$ mass of $0.1 M_{\odot}$. For the magnetar source, values of $E_{\text {rot }}=10$ foe and $t_{p}=1 \mathrm{~d}$ were used. It is clear that in the presence of a magnetar, the plateau luminosity and duration can change substantially. Also, differences in the phospheric velocity evolution are notable. Models with magnetars produce higher velocities. An interesting feature of magnetar models is the existence of a short phase of increasing luminosity preceding the plateau phase. This rise can be as large as one order of magnitude, which is much greater and steeper than in the case without a magnetar. If a $\mathrm{SN}$ is discovered early enough, this feature of the magnetar models can help to distinguish the power source.

For a deeper comparison between the models presented in Fig. 1, the profiles of different physical quantities (velocity, density, and temperature) at some specific epochs after the explosion are shown in Fig. 2. The most notable differences are in the velocity profiles. Almost the entire ejecta reach very high velocities in the presence of a magnetar. This explains the differences in the photospheric velocities seen in lower panel of Fig. 1. It is interesting to note that homologous expansion is reached around four days after the explosion for the model without a magnetar whereas it is delayed until around 50 days for the magnetar model. This means that the ejecta dynamics is modified after the shock break-out by the extra magnetar-powered force. As a result, the inner density of the ejecta becomes extremely low at the final phases of the simulation, while most of the ejected matter $\left(\sim 10 M_{\odot}\right)$ moves with speed $\sim 10^{4} \mathrm{~km} \mathrm{~s}^{-1}$. Figure 3 shows the radial distribution of the mass density into the ejecta. We note a thin, more dense shell is formed as the supernova expands without any opposing pressure outside, therefore starting to create a large bubble. The found overall behavior is consistent with the 2D-simulations by Chen et al. (2016) who pointed out that instabilies arise from the piling up of radiatively accelerated matter. We can guess that the full trapping of the magnetar power and the $1 \mathrm{D}$ approch are questionable, but a stronger statement is beyond the purpose of the present study.

We define some quantitative parameters which can help to characterize and compare LC morphologies. We call $L_{\max }$ the mean value of the local maxima produced after the shock peak,

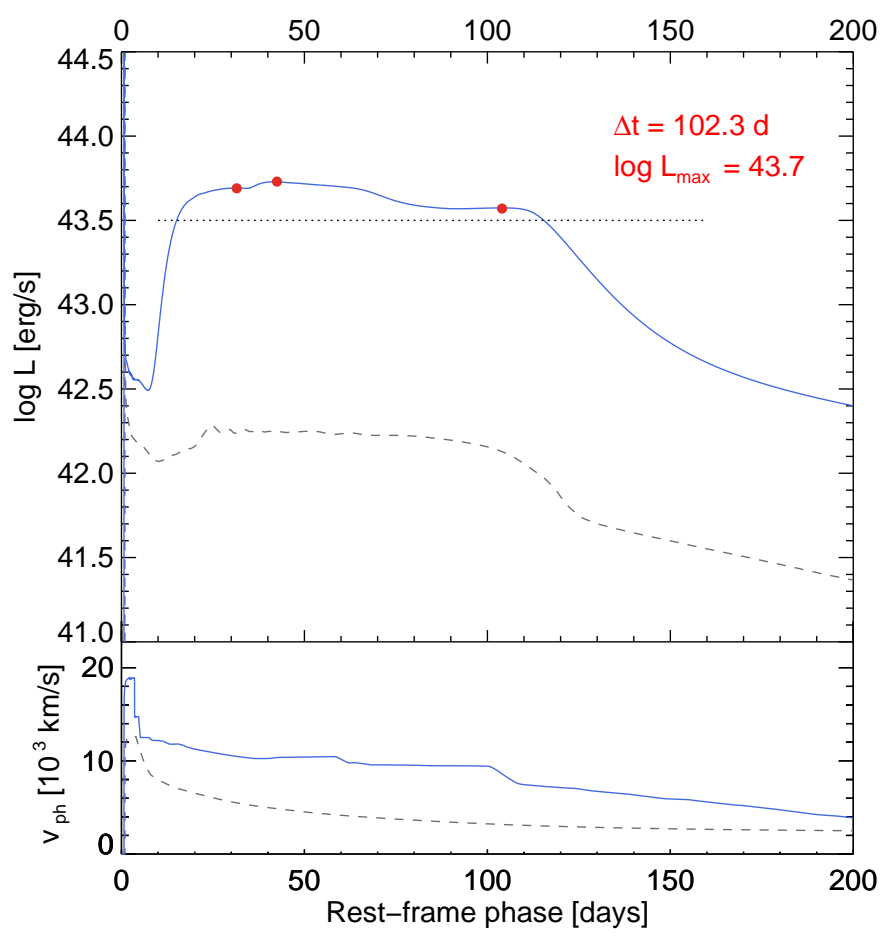

Fig. 1. Light-curve (top panel) and photospheric velocities (bottom panel) for our reference model (see text) shown in blue solid line, corresponding to a magnetar with $E_{\mathrm{rot}}=10$ foe and $t_{p}=1 \mathrm{~d}$. A characteristic maximum luminosity $L_{\max }$ is derived as the mean of the three local maxima found in the LC (red dots). The intersection of the horizontal line defined as $\log L_{\max }-0.2$ dex, with the LC provides the estimated temporal extent of that maximum, $\Delta t$. For comparison, we show the same SN model without a magnetar with dashed gray lines.

as illustrated in Fig. 1. In some cases, only one clear maximum is obtained. To characterize the temporal extent of the LC we measure the interval $\Delta t$ over which $\log L>\left(\log L_{\max }-0.2 \mathrm{dex}\right)$. The value of 0.2 dex in our definition is motivated by Bersten (2013) and references therein. These parameters are similar to the plateau luminosity and duration in the cases resembling Type II-P SNe. In this regard, we note that there is a variety of similar quantities defined elsewhere in the literature. A recent discussion on the duration-luminosity phase space of optical transients by Villar et al. (2017) applies a somewhat similar definition to ours, whereas the observational treatment proposed by Olivares (2010) is only applicable if a plateau phase and a clear transition from the plateau to the decline tail ${ }^{1}$ can be traced. In the investigation of magnetar-powered ordinary Type II-P SNe Sukhbold \& Thompson (2017), the plateau duration is measured in a very different way - those authors use the time from the explosion until the moment when the photospheric radius falls below $10^{14} \mathrm{~cm}$. This definition, although useful from the theoretical point of view, is not directly measurable in observations. In the following section we measure the parameters defined here $\left(L_{\max }, \Delta t\right)$ for a set of magnetar parameters.

\subsection{Grid of models}

We have calculated a set of SN LC models for different values of $t_{p}$ and $E_{\text {rot }}$. The considered values are intended to cover as

\footnotetext{
1 A transition is present in cases that we call intermediate but probably the nebular phase deserves a more careful treatment than our simplified analysis.
} 

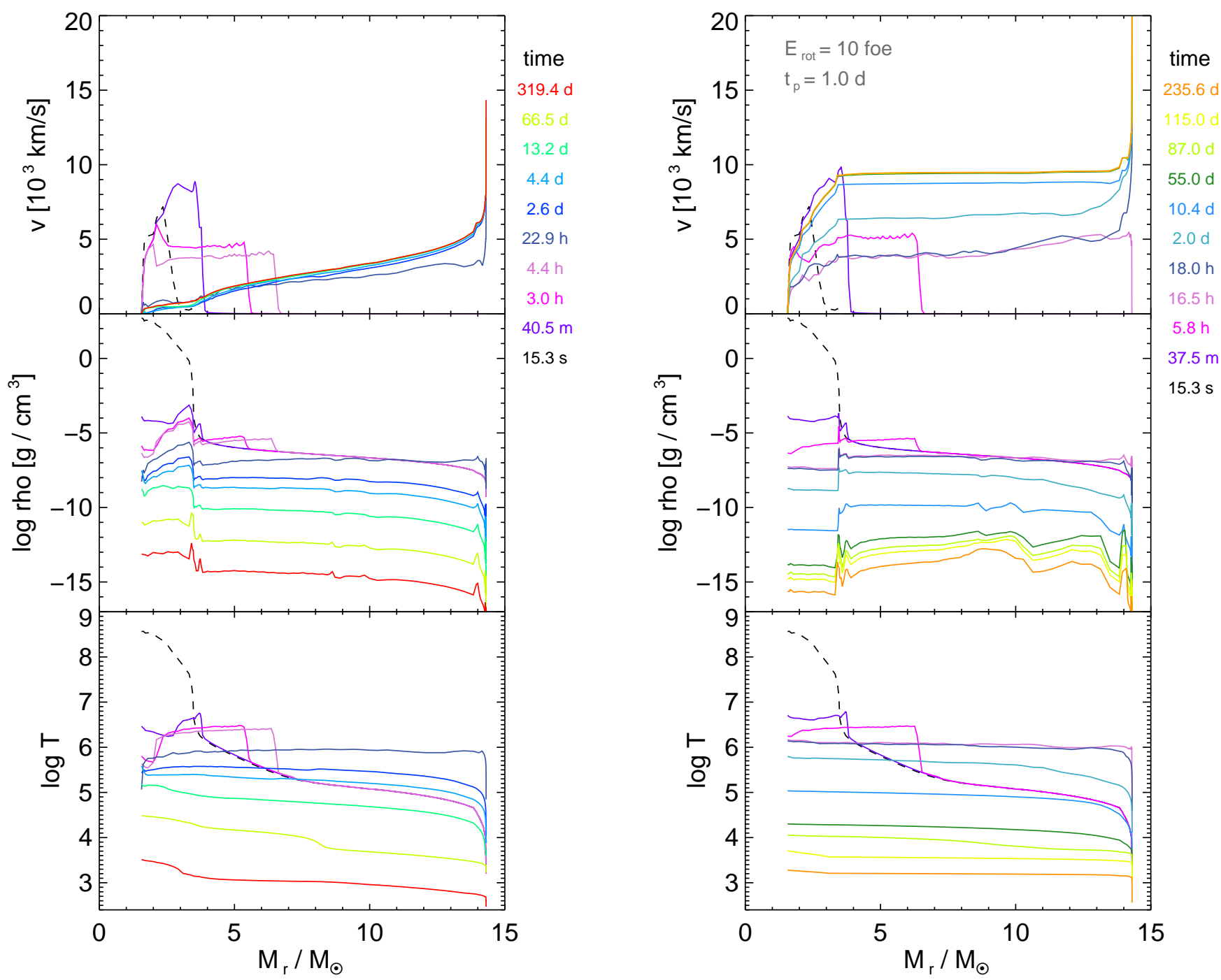

Fig. 2. Effect of the magnetar on the velocity, density and temperature profiles, as a function of the Lagrangian mass coordinate. Right panels: same pre-SN model as on the left but including a magnetar with $E_{\mathrm{rot}}=10$ foe and $t_{p}=1 \mathrm{~d}$, and all other parameters fixed. The color code of the epochs is preserved in each column, and is measured starting at the moment of $E_{\mathrm{k}}$ injection. With this powerful magnetar (right panels) the maximum velocity reaches one third of the speed of light at the edge of the ejecta. We cut the velocity axis in order to show the most relevant interval.

much of the parameters range as possible for the progenitor previously described. We have assumed a standard explosion energy $\left(E_{\mathrm{k}}=1.5 \times 10^{51} \mathrm{erg}\right)$ and ${ }^{56} \mathrm{Ni}$ production $\left(0.1 M_{\odot}\right)$. In Table 1 we provide information of the magnetar parameters for the grid of simulations performed here, together with the LC parameters as defined in the previous seccion $\left(L_{\max }\right.$ and $\left.\Delta t\right)$. Metzger et al. (2015) demonstrate that the maximum available rotational energy (without accounting for gravitational waves) of a NS is in the range of $90-165$ foe. Here we restrict to $E_{\text {rot }}=1-100$ foe, and $t_{p}=0.03-10$ days. The two most extreme conditions, $t_{p}=$ 10 days combined with $E_{\text {rot }}=30$ and 100 foe, respectively, were not capable to run with the same configuration as the others, so we choose not to include them here.

Figure 4 illustrates the distinct kinds of LC morphologies that we obtained. Some LCs present a well-defined peak, while others show a plateau phase. There are intermediate cases that show a slow decrease after the peak and a later break in the decline slope at the transition to the tail. The late-time slope at $t>200$ days is determined by the competing magnetar energy supply (Eq. (1)) and the $\mathrm{Ni}-\mathrm{Co}-\mathrm{Fe}$ radioactive deposition power.
Regarding the expansion of the ejecta, in Fig. 5 we present the model photospheric velocities. We note that the photospheric velocities seem to be more dependent on $E_{\text {rot }}$ than on $t_{p}$. However, this is not easy to connect with the kinetic energy of the ejecta due to the important effect of recombination on the photospheric velocities; although see Wang et al. (2016) for an alternative analytical treatment of the energetics. Our results show that larger values of $E_{\text {rot }}$ produce larger photospheric velocities, in other words a more important dynamical effect. In some cases, the expansion leads to an increase in the photospheric velocity during some time, as has been observed, for instance, in the peculiar SN 2005bf (Folatelli et al. 2006).

When considering the integrated luminosities during the whole SN evolution, models with low $E_{\text {rot }}$ are more efficient in converting the magnetar energy into radiation. Models with $E_{\mathrm{rot}}=1$ foe can radiate up to a third of the energy injected by the magnetar, while for $E_{\mathrm{rot}}=100$ foe, this efficiency is $\lesssim 2 \%$.

For low $E_{\text {rot }}$, though the dynamics of the ejecta seems less affected (see Fig. 5) the velocities during the plateau phase are systematically larger than in the case without a magnetar. With 


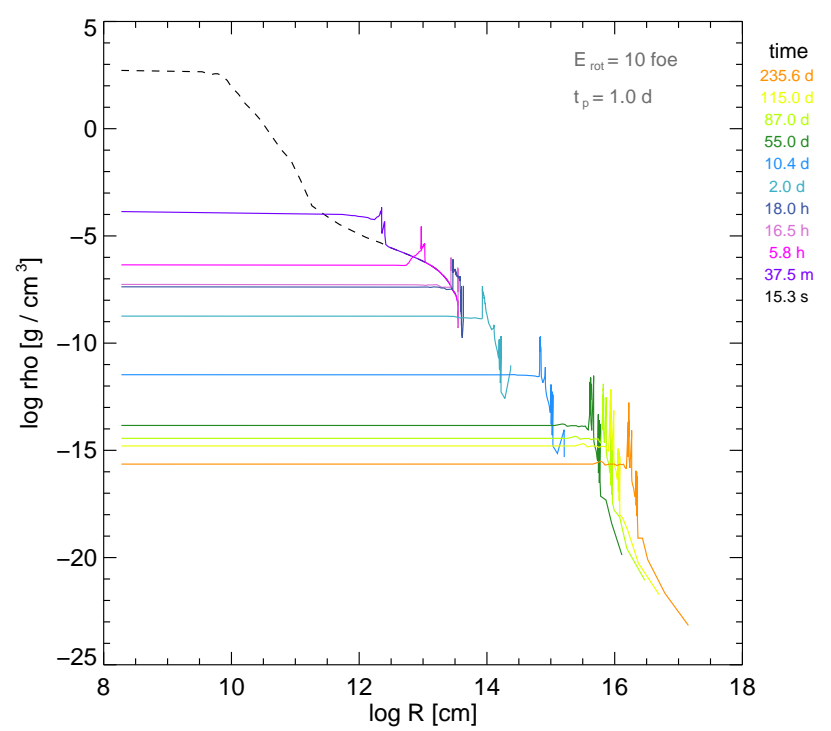

Fig. 3. Density profiles as function of the radius for the same magnetar of Fig. 1 and epochs as in Fig. 2.

increasing $E_{\mathrm{rot}}$ more is energy available, thus the photosphere gets larger velocities at earlier times. Hence the ejecta dilute before, so the nebular phase might be reached earlier (left and medium panels of Fig. 5). For a fixed $E_{\text {rot }}$, the photospheric velocities evolve faster for decreasing $t_{p}$.

Light-curve observables such as $L_{\max }$ and $\Delta t$, and their relation with the magnetar parameters are shown in Fig. 6. This figure can be used to obtain a rapid first guess of the magnetar parameters that may reproduce an observed $\mathrm{SN}$, as well as to understand the dependence of some observables with the magnetar parameters. We note that a similar analysis was done by Kasen \& Bildsten (2010) but for $\mathrm{H}$-free progenitors and using a different parameterization of the magnetar properties. Interestingly, for the cases having a plateau-like LC without a single peak, the duration $\Delta t$ resembles the plateau duration in ordinary SNe II-P, with a mean value $\Delta t \sim 80$ days, and extending up to $\sim 140$ days.

On the other extreme, a few of our single-peak LCs would be considered bright and rapidly evolving transients. These are usually the cases with very large $E_{\text {rot }}$. The cases with low values of $E_{\text {rot }}$ have peak luminosities below $\simeq 10^{43} \mathrm{erg} \mathrm{s}^{-1}$ and would not be called SLSNe. This is more evident in Fig. 7, which suggests that for the parameter space sampled here, there is a correlation between $L_{\max }$ and $E_{\text {rot }}$ with a scatter inversely proportional to $E_{\text {rot }}$. Figure 7 is also useful to visualize the individual values of the parameters used in our exploration.

\section{Application to observed $\mathrm{SNe}$}

In order to test whether magnetar-powered H-rich $\mathrm{SNe}$ can be a viable explanation for some observed events, we have modeled the evolution of two peculiar H-rich SNe (OGLE14-073 and SN 2004em). Table 2 shows the model parameters used to model the objets discussed in this section.

\subsection{OGLE14-073}

The recently reported OGLE14-073 (Terreran et al. 2017) at $z=0.1225$ presented a bright and very broad LC. Its spectra show prominent P-Cygni features of hydrogen but no sign of
Table 1. Model parameters and main characteristics of the magnetar (period, magnetic field strength) for the $15 M_{\odot}$ RSG stellar progenitor.

\begin{tabular}{|c|c|c|c|c|c|c|}
\hline Mod. & $\begin{array}{l}t_{p} \\
(\mathrm{~d})\end{array}$ & $\begin{array}{c}E_{\text {rot }} \\
\text { (foe) }\end{array}$ & $\begin{array}{c}P^{a} \\
(\mathrm{~ms})\end{array}$ & $\begin{array}{c}B^{a} \\
\left(10^{14} \mathrm{G}\right)\end{array}$ & $\begin{array}{l}\log L_{\text {max }} \\
\left(\mathrm{erg} \mathrm{s}^{-1}\right)\end{array}$ & $\begin{array}{l}\Delta t \\
\text { (d) }\end{array}$ \\
\hline 0 & - & - & - & - & 42.25 & 100.3 \\
\hline 1 & 0.03 & 1.0 & 5.07 & 72.60 & 42.47 & 87.8 \\
\hline 2 & 0.03 & 3.0 & 2.92 & 41.91 & 42.73 & 73.8 \\
\hline 3 & 0.03 & 10.0 & 1.60 & 22.96 & 43.15 & 57.5 \\
\hline 4 & 0.03 & 30.0 & 0.92 & 13.25 & 43.57 & 52.1 \\
\hline 5 & 0.03 & 100.0 & 0.51 & 7.26 & 43.99 & 38.5 \\
\hline 6 & 0.1 & 1.0 & 5.07 & 39.76 & 42.51 & 83.8 \\
\hline 7 & 0.1 & 3.0 & 2.92 & 22.96 & 42.88 & 67.8 \\
\hline 8 & 0.1 & 10.0 & 1.60 & 12.57 & 43.32 & 59.4 \\
\hline 9 & 0.1 & 30.0 & 0.92 & 7.26 & 43.78 & 61.6 \\
\hline 10 & 0.1 & 100.0 & 0.51 & 3.98 & 44.30 & 28.5 \\
\hline 11 & 0.1 & 300.0 & 0.29 & 2.30 & 44.72 & 12.1 \\
\hline 12 & 0.3 & 1.0 & 5.07 & 22.96 & 42.60 & 85.6 \\
\hline 13 & 0.3 & 3.0 & 2.92 & 13.25 & 43.04 & 70.5 \\
\hline 14 & 0.3 & 10.0 & 1.60 & 7.26 & 43.51 & 74.8 \\
\hline 15 & 0.3 & 30.0 & 0.92 & 4.19 & 44.00 & 48.0 \\
\hline 16 & 0.3 & 100.0 & 0.51 & 2.30 & 44.55 & 20.5 \\
\hline 17 & 1.0 & 1.0 & 5.07 & 12.57 & 42.86 & 78.8 \\
\hline 18 & 1.0 & 3.0 & 2.92 & 7.26 & 43.25 & 85.8 \\
\hline 19 & 1.0 & 10.0 & 1.60 & 3.98 & 43.67 & 102.3 \\
\hline 20 & 1.0 & 30.0 & 0.92 & 2.30 & 44.21 & 42.2 \\
\hline 21 & 1.0 & 100.0 & 0.51 & 1.26 & 44.68 & 16.5 \\
\hline 22 & 3.0 & 1.0 & 5.07 & 7.26 & 43.06 & 88.6 \\
\hline 23 & 3.0 & 3.0 & 2.92 & 4.19 & 43.44 & 108.9 \\
\hline 24 & 3.0 & 10.0 & 1.60 & 2.30 & 43.88 & 77.7 \\
\hline 25 & 3.0 & 30.0 & 0.92 & 1.33 & 44.29 & 43.8 \\
\hline 26 & 3.0 & 100.0 & 0.51 & 0.73 & 44.69 & 18.1 \\
\hline 27 & 10.0 & 1.0 & 5.07 & 3.98 & 43.23 & 104.4 \\
\hline 28 & 10.0 & 3.0 & 2.92 & 2.30 & 43.55 & 138.6 \\
\hline 29 & 10.0 & 10.0 & 1.60 & 1.26 & 44.00 & 74.4 \\
\hline 30 & 30.0 & 1.0 & 5.07 & 2.30 & 43.33 & 123.4 \\
\hline 31 & 30.0 & 3.0 & 2.92 & 1.33 & 43.68 & 102.0 \\
\hline
\end{tabular}

Notes. Each model has fixed $t_{p}$ and $E_{\mathrm{rot}}$. The observables from the light curve $\left(L_{\max }\right.$ and $\left.\Delta t\right)$ result from our numerical simulations. ${ }^{(a)}$ Assuming $I=1.3 \times 10^{45} \mathrm{~g} \mathrm{~cm}^{2}$, and $R=10 \mathrm{~km}$ for the NS.

interaction with a CSM. The slow spectrophotometric evolution for OGLE14-073 is consistent with a classification as a peculiar Type II event, similar to SN 1987A but much brighter. The explosion date of OGLE14-073 is not well constrained. Large values for the explosion energy $(\sim 12$ foe $)$ and ejecta mass $\left(\sim 60 M_{\odot}\right)$ as well as a rather large ${ }^{56} \mathrm{Ni}$ mass $\left(>0.47 M_{\odot}\right)$ need to be invoked in order to match the maximum luminosity and the late decline. The extreme values required to explain the properties of this object indicate that possibly another source is responsible for its brightness. Terreran et al. (2017) presented a magnetar as a viable explanation and discussed alternative scenarios for this event. Recently, Dessart \& Audit (2018) modeled this object as magnetar-powered. A discussion comparing this work and ours is presented in Sect. 5. In a different proposal, Moriya et al. (2018) studied OGLE14-073 as a possible fallback accretionpowered SN following a failed explosion of a massive star.

We first conducted an exploratory analysis via $\chi^{2}$ minimization over the set of LC models presented in the previous section. From the derived tentative values, and based on the experience with other SNe, we decided to vary the mass of the progenitor. 

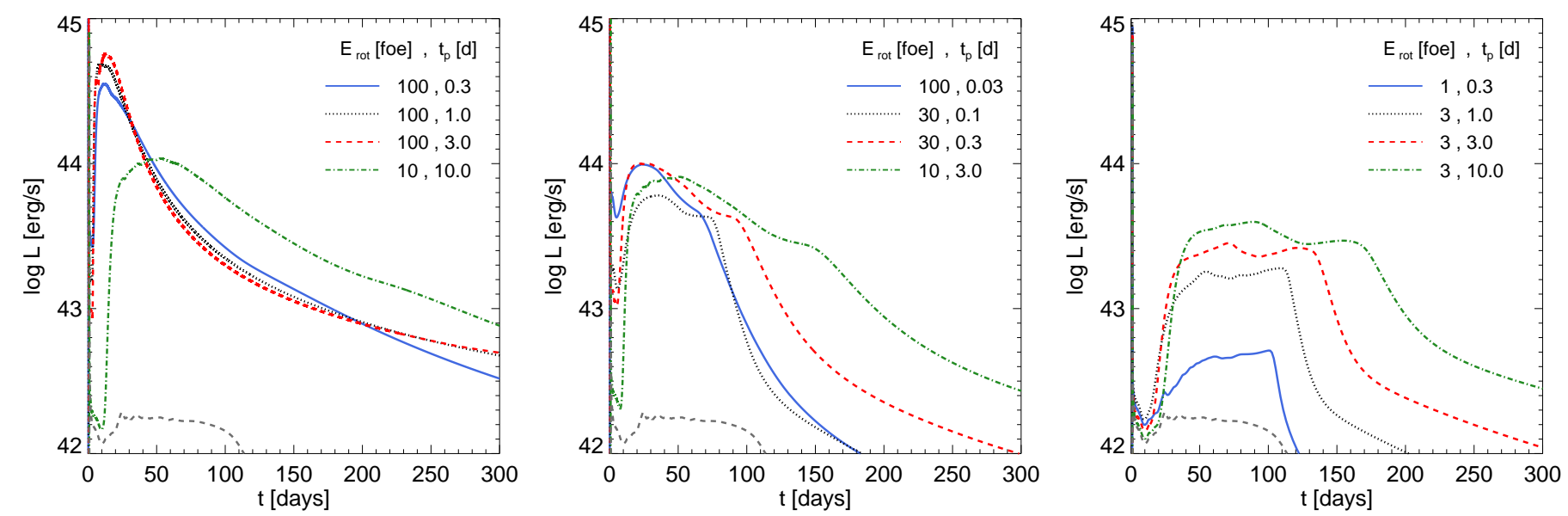

Fig. 4. Representative examples of the computed LC set. Left panel: LCs that have one clear peak, central panel: slower declining LCs (or intermediate cases) usually presenting a broken evolution in the slope, and right panel: cases with a plateau, that is, bright Type II-P. Legends indicate the parameters $E_{\mathrm{rot}}$ in units of $10^{51} \mathrm{erg}$, and $t_{p}$ in days. For comparison we include in dashed gray line the LC of the same SN without a magnetar.
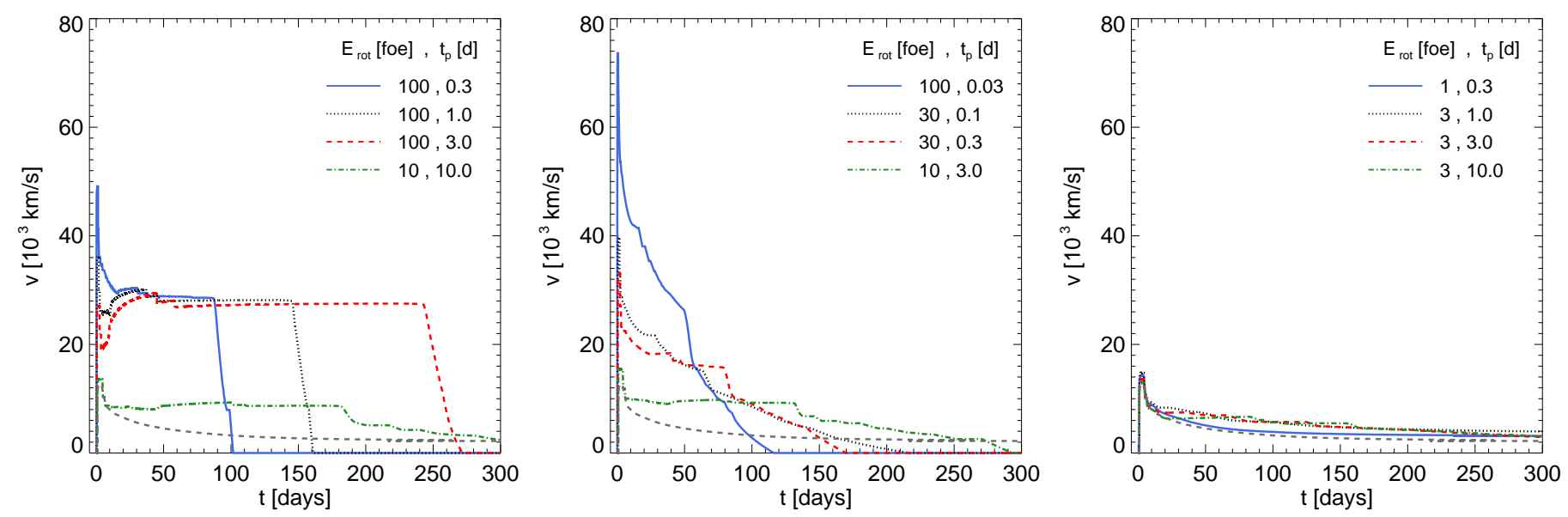

Fig. 5. Photospheric expansion velocity for the examples of Fig. 4. This velocity is null when the photosphere reaches the NS surface, that is, when the entire ejecta becomes transparent. Legends as in Fig. 4

Our preferred LC is presented in Fig. 8 and the model parameters are given in Table 2. This simulation was obtained with a main-sequence mass progenitor of $25 M_{\odot}$ which has a $\simeq 7 M_{\odot}$ He-rich core and $R \simeq 1200 R_{\odot}$ (see Appendix $\mathrm{B}$ for details on the chemical abundances). The explosion was initiated by a thermal bomb that released an energy of 2 foe. We assumed $0.2 M_{\odot}$ of ${ }^{56} \mathrm{Ni}$ and explored the parameters of the magnetar around the values obtained from our lower-mass models. As shown in Fig. 8 the LC data up to $\sim 200$ days is reasonably well fitted by a magnetar with $t_{p}=3$ days, and $E_{\mathrm{rot}}=0.8 \times 10^{51} \mathrm{erg}$. We assumed an interval of 20 days between the explosion and the first observed data. We note that we used the explosion date as a free parameter of the fit, only limited by the date of last non-detection, which occurred around 100 days before discovery (Terreran et al. 2017). For completeness, a comparison between our model photospheric velocities and the measured Fe II line velocities is also presented in Fig. 8. The model underestimates the iron velocities at early times, which could indicate either that a different progenitor or a slightly more powerful magnetar could be required. However, we note that although iron velocities are usually adopted as tracers of the photospheric velocity for normal SNe (see e.g., Takáts \& Vinkó 2012), this has not been fully proven for magnetar-powered SNe II.

\section{2. $S N 2004 e m$}

Another interesting case is SN $2004 \mathrm{em}$, the most extreme member of a small group of slowly rising Type II SNe (Taddia et al. 2016). Arcavi et al. (2012) commented on the peculiar photometric behavior of SN 2004em. For the first few weeks it was similar to a Type II-P SN, while at around day 25 it suddenly changed behavior to resemble a SN 1987A-like event, with similar long LC rise and expansion velocities. The total rise time was $\sim 110$ days, and only few additional observations were carried after the LC maximum. Although it was not as bright as SLSNe, Taddia et al. (2016) modeled the LC with a rather large kinetic energy, $E_{\mathrm{k}}=11.3$ foe and estimated $M_{\mathrm{ej}} \simeq 43 M_{\odot}$. Both kinetic energy and ejecta mass are the largest in their sample of long-rising SNe II, a rare family with only six members identified at the time ${ }^{2}$. The extreme values of the physical parameters needed to model this object may be an indication that this $\mathrm{SN}$ may have been powered by other mechanisms. The radius of the progenitor and the degree of nickel mixing in Taddia et al. (2016) were derived from hydrodynamical modeling done with the SuperNova Explosion Code (SNEC) and based on progeni-

2 According to a later poster there were eight members, see http: //sn2016.cl/documents/posters/poster_taddia.pdf 




Fig. 6. Main characteristics of the resulting LCs for a $15 M_{\odot}$ RSG progenitor. The color scale indicates the maximum LC luminosity after the shock breakout. Contours show the estimated temporal extent of the maximum (lines of constant $\Delta t$, see Fig. 1). The smallest, unlabeled contour corresponds to 100 days.

tor stars constructed using MESA (Paxton et al. 2011) with radii of $320-350 R_{\odot}$ and nickel mixing of $25 \%$ in the inner layers. Taddia et al. (2016) also inferred a value of $M\left({ }^{56} \mathrm{Ni}\right) \simeq 0.1 M_{\odot}$ from the tail of the LC.

We performed a tentative fit to the LC of SN 2004em. Our main goal was to see if we can approximately reproduce the observed rise and $L_{\max }$ assuming a magnetar power source. Our modeling procedure again started with a $\chi^{2}$ minimization using our grid of models for $15 M_{\odot}$ and $M\left({ }^{56} \mathrm{Ni}\right) \simeq 0.1 M_{\odot}$. The best fit was too bright, so we decreased the explosion energy to $E_{\mathrm{k}}=0.8$ foe, and the magnetar parameters were adjusted to $E_{\text {rot }}=0.07$ foe and $t_{p}=10$ days. With these parameters we could reproduce most of the LC (the slow rise plus broad maximum), however the observed decline during the early cooling phase was much more slower than the one shown by our models. In order to improve the match to the early LC we assumed that the star was surrounded by a diluted medium that is shocked by the SN ejecta. Such an interaction only modifies the early part of the LC. A good match with the data was obtained by assuming this CSM to be extended out to $\sim 3800 R_{\odot}$ and to contain a mass of $\approx 0.5 M_{\odot}$. This optimal model is shown in Fig. 9. Although the choice of model parameters was based on the LC alone, the photospheric velocity evolution compared to the Fe II line velocities is shown for completeness in Fig. 9. As in the case of OGLE14-073, the model velocities underestimate the iron line velocities.

\section{Comparison to other works}

Two recent works focus on the analysis of magnetar effects on type II SNe (Sukhbold \& Thompson 2017; Dessart \& Audit 2018). As here, the simulations in these works use a one-dimensional radiation-hydrodynamics code assuming gray

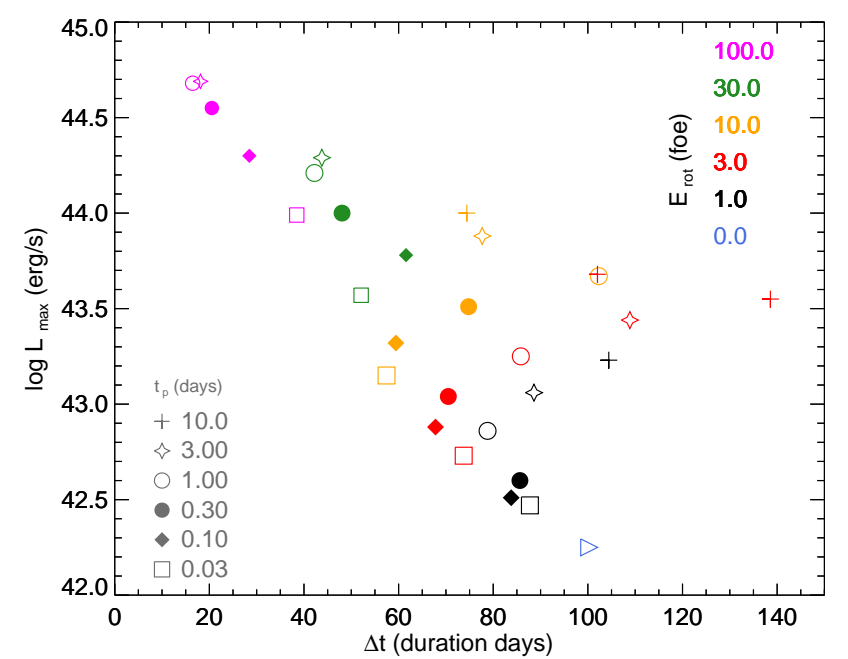

Fig. 7. Luminosity versus duration of the LCs. Each modeled LC is characterized by the rotational energy (color code) and spin-down time scale (symbols) of the magnetar, whereas all the other characteristics are fixed (see text). The triangle presents the model without a magnetar.

approximation for the radiation and including the magnetar source as an extra term in the energy equations assuming full energy trapping. The codes used in the two works were different, as well as the initial setup. For example, Dessart \& Audit (2018; hereafter DA18) used an Eulerian code (HERACLES) and they did not consider the radioactive decay.

In DA18 it is noticed that, in order to obtain density and temperature structures smooth at all times, an extended magnetar energy deposition is needed. Instead, here we have considered a limited range of mass where the magnetar energy is deposited. similar to Sukhbold \& Thompson (2017) prescription. The LCs of DA18 do not show a late time bump in the transition to the nebular phase. Such a bump is present in some of our cases, as well as in most of the LCs computed by Sukhbold \& Thompson (2017). Apart of this feature, the overall shapes of the LCs are similar. We note DA18 computed and discussed spectral features, while we made primary focus on the LCs. Regarding the fit to OGLE14-073, DA18 show two good matching models with ejected mass lower than ours $\left(M_{\mathrm{ej}}=11.9 M_{\odot}\right.$ and $M_{\mathrm{ej}}=17.8 M_{\odot}$ versus $M_{\mathrm{ej}} \sim 20.5 M_{\odot}$ in our case). They have obtained $E_{\mathrm{rot}}=0.4$ foe (versus $E_{\mathrm{rot}}=0.8$ foe) and $t_{p}=12 \mathrm{~d}$ that is similar to our $t_{p}=10 \mathrm{~d}$. Given the degeneracies of the problem, all these results seems to be consistent.

\section{Discussion and conclusions}

Magnetar-powered models generate a diversity of hydrogenrich SNe: ordinary and brighter ones. We have explored a wide range of magnetar properties by varying their rotational energies $E_{\mathrm{rot}}$ and spindown timescales $t_{p}$. If the commonly accepted values for the inertia moment $I=1.3 \times 10^{45} \mathrm{~g} \mathrm{~cm}^{2}$ and radius $R=10 \mathrm{~km}$ are adopted, then the inversion of the expressions relating magnetar parameters are $P \approx\left(5 / \sqrt{E_{\mathrm{rot}}}\right) \mathrm{ms}$, and $B \approx\left(1.25 \times 10^{15} / \sqrt{E_{\text {rot }} t_{p}}\right) \mathrm{G}$; with the energy in units of foe and the spindown timescale in days. This means that $t_{p}$ cannot be much smaller than our lowest value of 0.03 days if we want to keep the magnetic field strength of the NS comparable to those of known magnetars (see Olausen \& Kaspi 2014, for a reference about Galactic magnetars and their properties). 
Table 2. Summary of physical parameters for the SN progenitors used throughout this work.

\begin{tabular}{ccccccccccc}
\hline \hline & $M_{\text {ZAMS }}$ & $R$ & $M_{\mathrm{Ni}}$ & ${ }^{56} \mathrm{Ni}_{\text {mix }}$ & $X_{\text {sup }}$ & $Y_{\text {sup }}$ & $Z_{\text {sup }}$ & $E_{\mathrm{k}}($ foe $)$ & $E_{\text {rot }}($ foe $)$ & $t_{p}(\mathrm{~d})$ \\
\hline Grid & $15 M_{\odot}$ & $500 R_{\odot}$ & $0.1 M_{\odot}$ & 0.5 & 0.619 & 0.36 & 0.021 & 1.5 & Table 1 & 3 \\
OGLE14-073 & $25 M_{\odot}$ & $1200 R_{\odot}$ & $0.2 M_{\odot}$ & 0.95 & 0.573 & 0.408 & 0.019 & 2.0 & 0.8 & 3 \\
SN 2004em & $15 M_{\odot}^{*}$ & $500 R_{\odot}^{*}$ & $0.1 M_{\odot}$ & 0.5 & 0.619 & 0.36 & 0.021 & 0.8 & 0.07 & 10 \\
\hline
\end{tabular}

Notes. Values for the RSG configurations were obtained from stellar evolution calculations. The degree of ${ }^{56} \mathrm{Ni}$ mixing is given as a fraction of the interior mass of the model. The two rightmost columns provide the preferred magnetar parameters. ${ }^{*}$ Modified by adding $0.5 M_{\odot}$ of CSM extended out to $3800 R_{\odot}$.

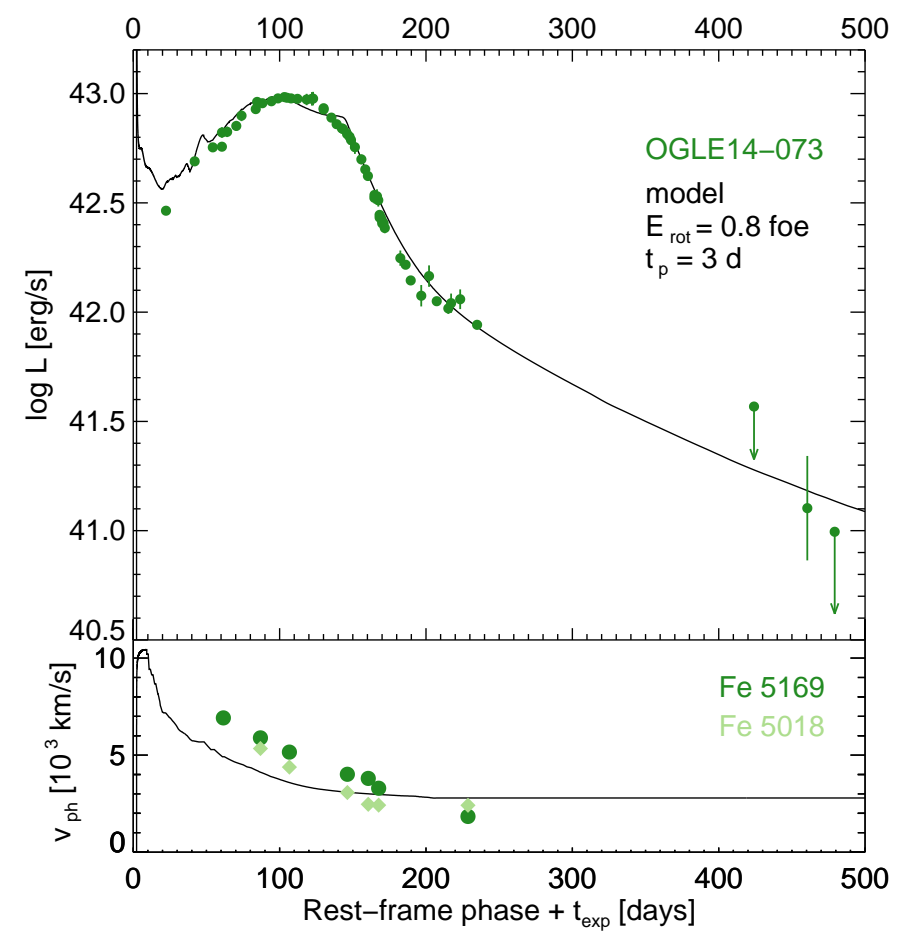

Fig. 8. Magnetar-powered SN model for OGLE14-073. The SN is assumed to have exploded $t_{\exp }=20$ days before discovery. The points show the bolometric luminosities (upper panel) and Fe II velocities (lower panel) from observations published by Terreran et al. (2017). The model is shown with solid lines and the parameters used in the simulation are presented in Table 2.

For a fixed progenitor mass of $15 M_{\odot}$ we found that magnetars spinning faster, but below physical breakup limit, produce more luminous events, being the spindown timescale related to the duration of the maximum brightness. Some combinations of the magnetar parameters produce a clear maximum in the LC followed by a smooth decline. In other cases, which we call intermediate LC morphologies, the declining slope breaks into a steeper tail. A third case shows a similar LC to those of normal Type II-P SNe (see the right panel of Fig. 4). Very bright Type II$\mathrm{P} \mathrm{SNe}$ are a distinctive class of events that have not been observed yet but can be produced by a magnetar source. A peculiar feature of these events is the existence of a phase when the luminosity increases by approximately one order of magnitude before the plateau is settled. In addition, the numerical experiments performed here led us to propose that some peculiar SN 1987Alike $\mathrm{SNe}$ can be explained by the magnetar source. Interestingly, we were able to produce the slowly rising SN-1987A-like LC morphology without assuming the usual BSG structure, which is also independently found by Sukhbold \& Thompson (2017).

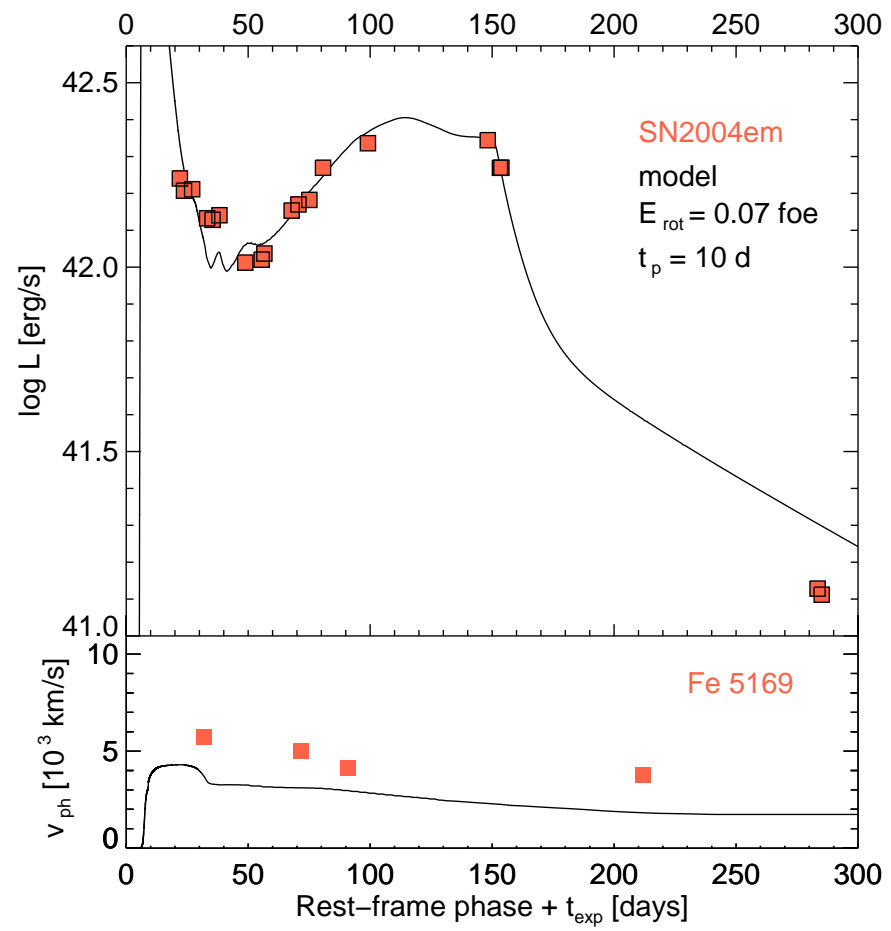

Fig. 9. Magnetar-powered model for SN 2004em. The SN is assumed to have exploded $t_{\exp }=10$ days before discovery. The points show the bolometric luminosities (upper panel) and Fe II velocities (lower panel) from observations published by Taddia et al. (2016). The model is shown with solid lines and the parameters used in the simulation are presented in Table 2. To reproduce the SN evolution during the cooling phase ( first month) an interaction between the SN ejecta and some circumstellar material was assumed (see more details in Sect. 4.2).

A summary of the magnetar and the stellar progenitor parameters is presented in Table 2, whereas the detailed chemical composition is given in Appendix B. We note that both ${ }^{56} \mathrm{Ni}$ and magnetar energy depositions were taken into account in our calculations. They relative influence depends on the specific values of the parameters adopted, as shown by Moriya et al. (2017).

We show that magnetar-powered explosion models can explain the overall luminosity of two observed H-rich SNe: the recent interesting case of OGLE14-073 (Terreran et al. 2017), and the mildly bright SN 2004em (Taddia et al. 2016). Our preferred model for OGLE14-073 has $P \sim 5 \mathrm{~ms}$ and $B \sim 7 \times 10^{14} \mathrm{G}$. For SN 2004em values of $P \sim 19 \mathrm{~ms}$ and $B \sim 1.5 \times 10^{15} \mathrm{G}$ are found based on the LC modelling around maximum. However, the presence of some CSM was needed in order to reproduce the early observations. In both cases, an RSG progenitor was assumed, with $M_{\text {ZAMS }}=15 M_{\odot}$ and $25 M_{\odot}$ for SN 2004em and OGLE14-073, respectively. 
The photospheric velocities of our models tend to lie below those measured from iron lines. Nevertheless, we were not focused on finding a model that reproduces both observables. Instead, our goal was to test whether a magnetar is able to reproduce the observed LC morphology of H-rich SNe. In any case, it is not clear if iron lines are an accurate tracer of the photospheric velocity in magnetar-powered objects, as is usually assumed for normal SNe II. Our models show that 1987A-like morphologies can be produced from RSG progenitors by including a magnetar source.

During the nebular phase our treatment is too simplified to expect a reliable match with the observations. This is, among other reasons, because the spectral energy distribution of the magnetar is not specified, whereas the bolometric data derived from observations usually assume thermal emission. Here we have adopted a braking index of $n=3$ (defined from $\dot{\Omega}=-k \Omega^{n}$ ) from dipolar radiation (Shapiro \& Teukolsky 1983), although a range of $1<n<2.8$ is observed in isolated pulsars. Therefore, a different exponent given by $-(n+1) /(n-1)$ is possible in the magnetar luminosity function (Eq. (1)), which allows for a steeper decline.

We conclude that the observational appearance of SNe II powered by magnetars is extremely varied. Future advances in the physics of this type of model will be very relevant.

Acknowledgements. This work was partially supported by grant PIUNRN2016-40B531. We are grateful to Omar Benvenuto for his valuable guidance in the development of the code and later modifications. We thank to G. Folatelli and to the anonymous referee for their help to improve the manuscript.

\section{References}

Akiyama, S., Wheeler, J. C., Meier, D. L., \& Lichtenstadt, I. 2003, ApJ, 584, 954

Arcavi, I., Gal-Yam, A., Cenko, S. B., et al. 2012, ApJ, 756, L30

Arcavi, I., Howell, D. A., Kasen, D., et al. 2017, Nature, 551, 210

Bersten, M. C. 2013, PhD Thesis, University of Chile

Bersten, M. C., \& Benvenuto, O. G. 2016, Bol. Asoc. Argent. Astron., 58, 246

Bersten, M. C., Benvenuto, O., \& Hamuy, M. 2011, ApJ, 729, 61

Bersten, M. C., Benvenuto, O. G., Orellana, M., \& Nomoto, K. 2016, ApJ, 817, L8

Burrows, A., Dessart, L., Livne, E., Ott, C. D., \& Murphy, J. 2007, ApJ, 664, 416 Chen, K.-J., Woosley, S. E., \& Sukhbold, T. 2016, ApJ, 832, 73

Chevalier, R. A., \& Irwin, C. M. 2011, ApJ, 729, L6

Dessart, L., \& Audit, E. 2018, A\&A, 613, A5
Drout, M. R., Chornock, R., Soderberg, A. M., et al. 2014, ApJ, 794, 23 Folatelli, G., Contreras, C., Phillips, M. M., et al. 2006, ApJ, 641, 1039 Gal-Yam, A. 2012, Science, 337, 927

Georgy, C., Ekström, S., Eggenberger, P., et al. 2013, A\&A, 558, A103

Gezari, S., Halpern, J. P., Grupe, D., et al. 2009, ApJ, 690, 1313

Heger, A., Fryer, C. L., Woosley, S. E., Langer, N., \& Hartmann, D. H. 2003, ApJ, 591, 288

Heger, A., Woosley, S. E., \& Spruit, H. C. 2005, ApJ, 626, 350

Hu, R.-Y., \& Lou, Y.-Q. 2009, MNRAS, 396, 878

Inserra, C., \& Smartt, S. J. 2014, ApJ, 796, 87

Inserra, C., Smartt, S. J., Jerkstrand, A., et al. 2013, ApJ, 770, 128

Inserra, C., Nicholl, M., Chen, T.-W., et al. 2017, MNRAS, 468, 4642

Inserra, C., Smartt, S. J., Gall, E. E. E., et al. 2018, MNRAS, 475, 1046

Janka, H.-T. 2012, Annu. Rev. Nucl. Part. Sci., 62, 407

Kasen, D., \& Bildsten, L. 2010, ApJ, 717, 245

Kasen, D., Metzger, B. D., \& Bildsten, L. 2016, ApJ, 821, 36

Maeda, K., Tanaka, M., Nomoto, K., et al. 2007, ApJ, 666, 1069

Metzger, B. D., Margalit, B., Kasen, D., \& Quataert, E. 2015, MNRAS, 454, 3311

Miller, A. A., Chornock, R., Perley, D. A., et al. 2009, ApJ, 690, 1303

Misner, C. W., \& Sharp, D. H. 1969, Quasars and High-energy Astronomy (New York: Gordon) 397

Moriya, T. J., Blinnikov, S. I., Tominaga, N., et al. 2013, MNRAS, 428, 1020

Moriya, T. J., Chen, T.-W., \& Langer, N. 2017, ApJ, 835, 177

Moriya, T. J., Terreran, G. \& Blinnikov, S. I. 2018, MNRAS, 475, L11

Mösta, P., Ott, C. D., Radice, D., et al. 2015, Nature, 528, 376

Nicholl, M., Smartt, S. J., Jerkstrand, A., et al. 2015, MNRAS, 452, 3869

Nicholl, M., Guillochon, J., \& Berger, E. 2017, ApJ, 850, 55

Nomoto, K., \& Hashimoto, M. 1988, Phys. Rep., 163, 13

Olausen, S. A., \& Kaspi, V. M. 2014, ApJS, 212, 6

Olivares, E., F., Hamuy, M., Pignata, G., , et al. 2010, ApJ, 715, 833

Paxton, B., Bildsten, L., Dotter, A., et al. 2011, ApJS, 192, 3

Quimby, R. M., Kulkarni, S. R., Kasliwal, M. M., et al. 2011, Nature, 474, 487

Shapiro, S. L., \& Teukolsky, S. A. 1983, Black Holes, White Dwarfs and

Neutron: The Physics of Compact Objects (New York: Wiley-Interscience), 663

Smith, N., Li, W., Foley, R. J., et al. 2007, ApJ, 666, 1116

Soker, N., \& Gilkis, A. 2017, ApJ, 851, 95

Sukhbold, T., \& Thompson, T. A. 2017, MNRAS, 472, 224

Sukhbold, T., \& Woosley, S. E. 2016, ApJ, 820, L38

Taddia, F., Sollerman, J., Fremling, C., et al. 2016, A\&A, 588, A5

Taddia, F., Sollerman, J.Fremling, C., et al. 2018, A\&A, 609, A106

Takáts, K., \& Vinkó, J. 2012, MNRAS, 419, 2783

Terreran, G., Pumo, M. L., Chen, T.-W., et al. 2017, Nat. Astron., 1, 713

Turolla, R., Zane, S., \& Watts, A. L. 2015, Rep. Progr. Phys., 78, 116901

van Riper, K. A. 1979, ApJ, 232, 558

Villar, V. A., Berger, E., Metzger, B. D., \& Guillochon, J. 2017, ApJ, 849, 70

Wang, L.-J., Wang, S. Q., Dai, Z. G., et al. 2016, ApJ, 821, 22

Wheeler, J. C., Yi, I., Höflich, P., \& Wang, L. 2000, ApJ, 537, 810

Woosley, S. E. 2010, ApJ, 719, L204

Yan, L., Lunnan, R., Perley, D. A., et al. 2017, ApJ, 848, 6

Yu, Y.-W., Zhu, J.-P., Li, S.-Z., Lü, H.-J., \& Zou, Y.-C. 2017, ApJ, 840, 12 


\section{Appendix A: Equations of relativistic radiating hydrodynamics}

The code we employed is a modified version of that described in Bersten et al. (2011). It is a one-dimensional Lagrangian code that solves explicitly hydrodynamic equations while it assumes an implicit strategy for energy conservation and flux limited radiative transport. Although it is usual to consider that Newtonian physics is adequate for computing light curves of supernovae, we find that powerful enough magnetars may force the expanding envelopes to move to speeds comparable to the speed of light. Evidently, the physics assumed in our code required revision to properly handle relativistic velocities. For this purpose we have adopted the scheme presented by van Riper (1979), who assumes that the object evolves adiabatically, an approximation certainly not suitable for the problem we face in this paper. In order to include the corrections to the radiative transfer together with conservation of energy we have adopted the description presented in Misner \& Sharp (1969). Here we detail the equations implemented in our SN light-curve code.

We assume a metric such as

$\mathrm{d} s^{2}=-\mathrm{e}^{2 \phi} c^{2} \mathrm{~d} t^{2}+\left(\frac{1}{\Gamma} \frac{\partial r}{\partial m}\right)^{2} \mathrm{~d} m^{2}+r^{2} \mathrm{~d} \Omega^{2}$,

where $\Omega$ is the solid angle and

$\Gamma^{2}=1+\left(\frac{U}{c}\right)^{2}-\frac{2 G \tilde{m}}{r c^{2}}$.

The gravitational mass $\tilde{m}$ is given by

$\tilde{m}(m)=\int_{0}^{m}\left(1+E / c^{2}\right) \Gamma \mathrm{d} m^{\prime}$.

The velocity $U$ is

$\frac{\partial r}{\partial t}=\mathrm{e}^{\phi} D_{t} r=\mathrm{e}^{\phi} U$.

The coefficient of the metric is given by

$\frac{\partial \phi}{\partial P}=-\frac{V}{w c^{2}}$,

where $w$ is the relativistic enthalpy, given by

$w=1+\frac{E+P V}{c^{2}}$.

At the stellar surface the coefficient of the metric is

$\mathrm{e}^{\phi_{\mathrm{s}}}=\Gamma_{\mathrm{s}}^{-1}\left(1-\frac{2 G \tilde{m}_{\mathrm{s}}}{r_{\mathrm{s}} c^{2}}\right)$.

The equation of motion of the fluid is

$\frac{\partial U}{\partial t}=\mathrm{e}^{\phi}\left(-\frac{4 \pi \Gamma r^{2}}{w} \frac{\partial P}{\partial m}-\frac{G \tilde{m}}{r^{2}}-\frac{4 \pi G r P}{c^{2}}.\right)$

The specific volume is

$V=\frac{1}{\Gamma} \frac{\partial}{\partial m}\left(\frac{4 \pi}{3} r^{3}\right)$

The radiative luminosity is given by

$L=-\left(4 \pi r^{2}\right)^{2} \frac{a c}{3 \kappa} \mathrm{e}^{-4 \phi} \frac{\partial}{\partial A}\left(\mathrm{e}^{4 \phi} T^{4}\right)$

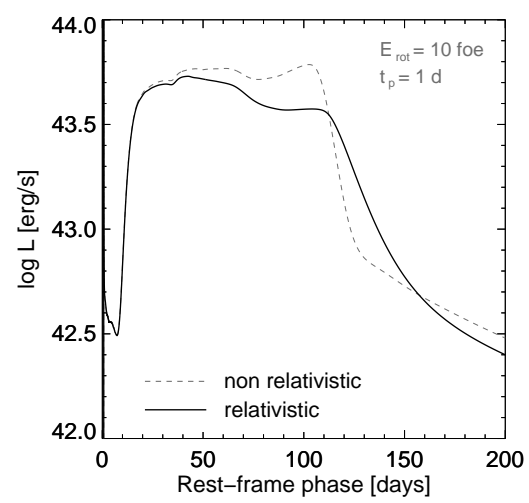

Fig. A.1. Light curves for the magnetar of Fig. 1.
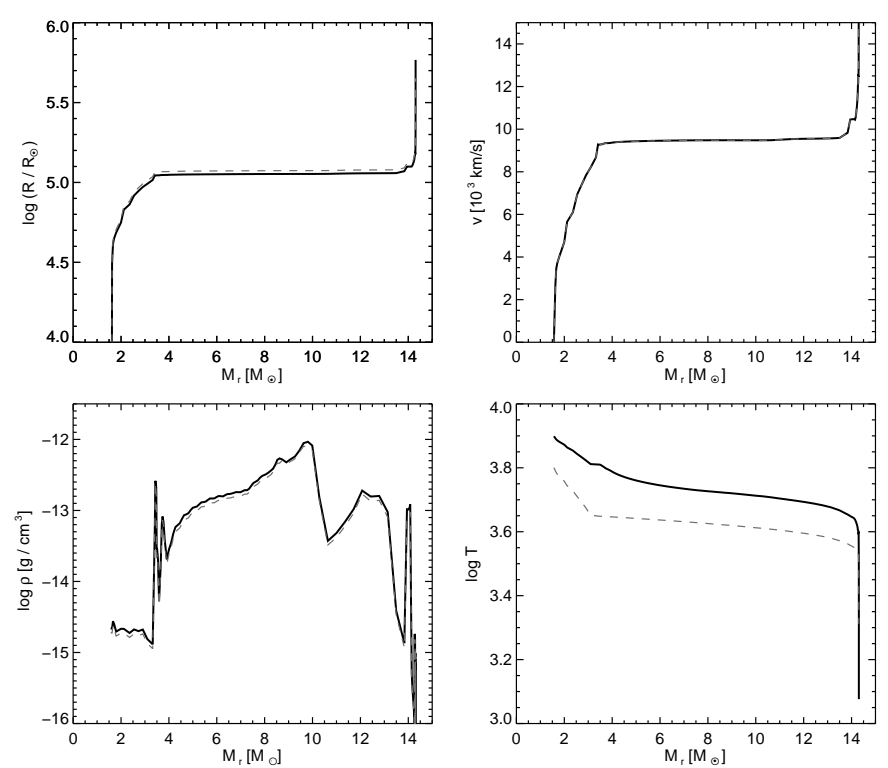

Fig. A.2. Inner physical quantities for the model with $E_{\mathrm{rot}}=10$ foe and $t_{p}=1 \mathrm{~d}$ commented in detail in the text. These are profiles at time $t=104$ days, when the niquel phase is ending and the photosphere receding. The continuous line corresponds to the relativistic solver and the dashed to the non-relativistic.

Finally, the equation of energy conservation is

$D_{t} E+P D_{t} V+\mathrm{e}^{-2 \phi} \frac{\partial}{\partial A}\left(\mathrm{e}^{2 \phi} L\right)=0$.

Results with the two solvers. As mentioned, the differences into calculations with the relativistic hydrodynamic detail are evident for powerful magnetars. Here we illustrate into Fig. A.1 the LCs obtained with Bersten et al. (2011) original treatment (plus magnetar) indicated as "non-relativistic" and with the present modified version as "relativistic" for the model presented in Fig. 1. With this powerful magnetar the LC is modified as result of the incorporation of the relativistic radiating hydrodynamics, although the overall morphology, according to our simple scheme, is preserved. We should classify as plateau-kind the LCs resulting with both solvers. However, a bump around $\sim 100 \mathrm{~d}$ is less prominent with the relativistic treatment. The profiles of density, radius, velocity and temperature for this model are presented in Fig. A.2. The most clear difference is noted in the temperature profile. If the temperature of the ejecta in the hydrogen rich layers changes around the temperature for hydrogen ionization this may substantially change the matter opacity and hence the outcoming luminosity. 


\section{Appendix B: Progenitor chemical composition}

We have considered RSG structures calculated by Nomoto \& Hashimoto (1988) as our SN progenitors. For completeness we provide their detailed composition in Figs. B.1 and B.2 for models with main-sequence masses of 15 and $25 M_{\odot}$ respectively. We note that the internal core is

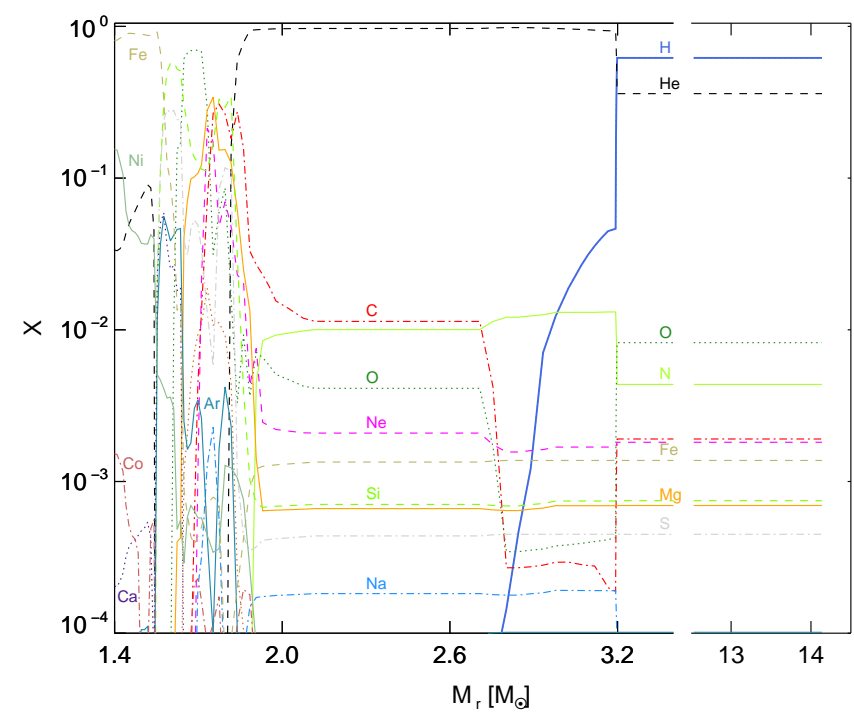

Fig. B.1. Chemical composition of a $M_{\mathrm{ZAMS}}=15 M_{\odot}$ star during the RSG state. removed for simplicity as it is considered to collapse and to form the magnetar. Chemical stratification presented is the result of stellar evolution calculations. However, as in other studies the ${ }^{56} \mathrm{Ni}$ distribution (modified by chemical mixing) was adapted for convenience and assuming a conservative value of $0.1 M_{\odot}$ in our grid calculations.

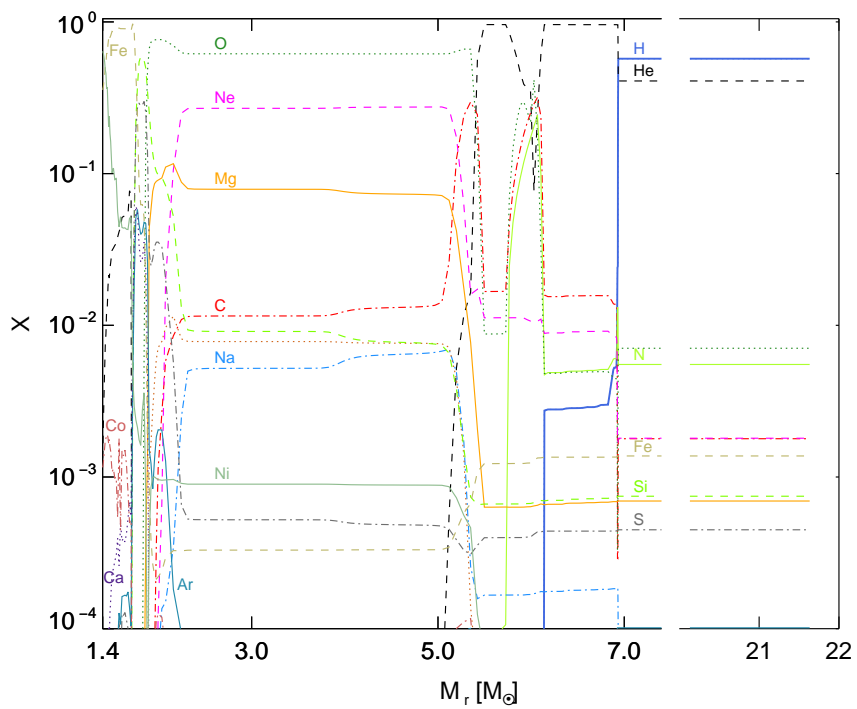

Fig. B.2. Chemical composition of a $M_{\text {ZAMS }}=25 M_{\odot}$ star during the RSG state. 